Additional services for Journal of Fluid Mechanics:

Email alerts: $\underline{\text { Click here }}$

Subscriptions: $\underline{\text { Click here }}$

Commercial reprints: $\underline{\text { Click here }}$

Terms of use : $\underline{\text { Click here }}$

\title{
Topographically forced long waves on a sheared coastal current. Part 2.
} Finite amplitude waves

S. R. CLARKE and E. R. JOHNSON

Journal of Fluid Mechanics / Volume 343 / July 1997, pp 153 - 168

DOI: 10.1017/S0022112097005697, Published online: 08 September 2000

Link to this article: http://journals.cambridge.org/abstract_S0022112097005697

How to cite this article:

S. R. CLARKE and E. R. JOHNSON (1997). Topographically forced long waves on a sheared coastal current. Part 2. Finite amplitude waves. Journal of Fluid Mechanics, 343, pp 153-168 doi:10.1017/S0022112097005697

Request Permissions : $\underline{\text { Click here }}$ 


\title{
Topographically forced long waves on a sheared coastal current. Part 2. Finite-amplitude waves
}

\author{
By S. R. CLARKE AND E. R. JOHNSON \\ Department of Mathematics, University College London, Gower St, London WC1E 6BT, UK
}

(Received 4 July 1996)

This paper analyses the finite-amplitude flow of a constant-vorticity current past coastal topography in the long-wave limit. A forced finite-amplitude long-wave equation is derived to describe the evolution of the vorticity interface. An analysis of this equation shows that three distinct near-critical regimes occur. In the first the upstream flow is restricted, with overturning of the vorticity interface for sufficiently large topography. In the second quasi-steady nonlinear waves form downstream of the topography with weak upstream influence. In the third regime the upstream rotational fluid is partially blocked. Blocking and overturning are enhanced at headlands with steep rear faces and decreased at headlands with steep forward faces. For certain parameter values both overturning and partially blocked solutions are possible and the long-time evolution is critically dependent on the initial conditions. The reduction of the problem to a one-dimensional nonlinear wave equation allows solutions to be followed to much longer times and parameter space to be explored more finely than in the related pioneering contour-dynamical integrations of Stern (1991).

\section{Introduction}

The method of contour dynamics (e.g. see Dritschel 1989) gives a particularly accurate and convenient method for studying flows with vorticity or potential vorticity interfaces. Stern (1991) used contour dynamics to study the evolution of a sheared coastal current passing a semicircular cape. The steady theories of Hughes (1985a, $b$, $1986 a, b, 1987,1989)$ and the unsteady weakly nonlinear theory of Grimshaw (1987) would suggest that some evidence of hydraulic control of the current should be exhibited by this system. These solutions should be a function of only the height of the topography at any point, with subcritical flow upstream of the topography and supercritical flow downstream. Gill (1977) proposed a formalism showing that hydraulically controlled rotating flows occur where one of the long-wave modes of the system is stationary, and at this point two different solutions can merge smoothly. Stern showed in the particular case of a uniformly sheared coastal current, the upstream flow can be blocked and downstream the flow resembles hydraulically controlled supercritical flow. Unlike hydraulic control no bore propagates upstream to create subcritical flow; rather, a localized wave appears to form at the upstream edge of the cape. Stern concluded that the blocking of the upstream flow was due to weak-hydraulic effects and was only possible when long waves could propagate upstream.

The weakly nonlinear long-wave limit of the flow of a sheared current past coastal topography is considered in the companion paper (Clarke \& Johnson 1997a, hereinafter referred to as Part 1), corresponding to the flow considered by Stern (1991) 
with the semicircular cape replaced by smooth topography. It is shown that steady hydraulically controlled solutions do not exist for this flow geometry, as the subcritical upstream flow and supercritical downstream flow cannot merge smoothly as required by Gill's theory. The evolution of the vorticity interface is shown to be governed by a forced version of the BDA (Benjamin-Davis-Acrivos) equation. An analysis of this equation in the near-critical limit shows the presence of two distinct near-critical regimes: one where the current narrows downstream and waves propagate upstream, and a second where steady periodic nonlinear waves form downstream, with weak upstream influence. In the hydraulic limit it is shown that these two regimes vanish, agreeing with Stern's conclusion that the blocking of the upstream sheared current is due to weak-hydraulic effects.

In a few special cases such as Haynes, Johnson and Hurst (1993), where only one wave mode is possible, or Grimshaw \& Yi (1992), where the interaction between a resonant mode and other modes is weak, unsteady finite-amplitude longwave theories can be used to study how hydraulic control by topography evolves. Grimshaw \& Yi (1990) demonstrated that in the long-wave limit the evolution of the interface between a infinite coastal strip of constant potential vorticity and an infinite outer region of constant, but differing, potential vorticity is governed by a finite-amplitude generalization of the BDA equation. Stern (1991) and Part 1 add coastal capes to this geometry (but note that in Part 1 the outer region is irrotational, while in Stern's problem the potential vorticity of the outer region is allowed to vary).

This paper extends the analysis of Grimshaw \& Yi (1990) to describe the evolution of a vorticity interface under the forcing of coastal topography. The resulting equation is then used to study and extend the analysis of Stern (1991) and Part 1. The advantage of the present analysis over Stern's pioneering contour-dynamical integrations lies in the efficiency of accurate integrations of the governing equations. This allows solutions to be carried to times twenty or so times greater than the contour-dynamical integrations so that the asymptotic behaviour of the flow becomes clear. The speed of these integrations also allows the parameter space to be more finely explored to produce a regime diagram unifying and classifying the various forms of the asymptotic flows. Particular attention is paid to the extent of blocking of the upstream flow and overturning of the interface. This flow and geometry is one of the simplest forms of coastal current that could be considered. As noted in Part 1 conjugate flow pairs exist with a wide subcritical reversed flow paired with a narrow unidirectional supercritical flow. In the hydraulic limit there is however no smooth transition possible between the conjugate solutions and the question arises as to what form the flow takes in the long-wave limit. It was shown in Part 1 that the weakly nonlinear near-critical flow remains unsteady in the neighbourhood of the coastal outcrop although it exhibits many of the characteristics of the steady conjugate flows. The present paper extends these results to finite amplitude. Other, almost as simple, geometries and depth profiles with piecewise-constant vorticity can exhibit smooth changes from subcritical to supercritical flow with hydraulic control at topographic extrema. An example is the rotating channel flow discussed in Haynes et al. (1993). It is the offshore unboundedness of the present flow that precludes a smooth transition.

Section 2 derives the appropriate forced finite-amplitude evolution equation for the vorticity interface following Grimshaw \& Yi (1990). The flow regimes for the flow past a symmetric obstacle are then discussed in $\S 3$, and the sensitivity of these solutions is considered in $\S \S 4$ and 5. The results of Stern (1991) and Part 1 suggest that the blocking is a non-local effect: the flow is not determined by the local topographic perturbation alone but is affected by the overall shape of the topography. Section 
Topographically forced long waves on a sheared coastal current. Part 2

155

4 shows that flow past forward- and backward-facing obstacles differs considerably from that past symmetric obstacles. Section 5 demonstrates the effect of differing the initial conditions and $\$ 6$ discusses the results.

\section{The finite-amplitude evolution equation}

The geometry of the flow to be considered is described in Part 1. An inviscid sheared constant-depth current flows past coastal topography. The flow is bounded by a rigid upper surface so the governing equation is the two-dimensional conservation of vorticity. The current has undisturbed width $H$ and constant vorticity $-Q$ (with $Q>0$ ). Offshore the flow is irrotational, approaching a uniform along-coast flow $U Q H$ at large distances from the coast. The Cartesian coordinates $x$ (along-shore) and $y$ (offshore) are scaled on $H$, time $t$ on $Q^{-1}$ and velocities on $Q H$. The outcropping topography along the vertical coastal wall, $f(x)$, satisfies

$$
\lim _{|x| \rightarrow \infty} f=0 .
$$

The interface between the sheared coastal current and the irrotational outer flow is $h(x, t)$. As this interface is a material surface

$$
h_{t}=\frac{\mathrm{d}}{\mathrm{d} x}\{\psi(x, h, t)\},
$$

where $\psi(x, y, t)$ is the dimensionless streamfunction. The topography has a characteristic dimensionless lengthscale $\mu^{-1}$ and is assumed to be long compared to the width of the shear layer, so $\mu \ll 1$. Long length- and timescales are introduced as

$$
\chi=\mu x, \quad T=\mu t .
$$

In the inner region, $y<h(\chi, T)$, as shown in Part $1, \psi$ takes the form

$$
\psi=-\frac{1}{2}(y-f)^{2}+C(\chi, T)(y-f)+O\left(\mu^{2}\right),
$$

where $C(\chi, t)$ is the, as yet undetermined, speed at the coast. In the outer region, $y>h(\chi, T)$,

$$
\psi=-U y+\frac{1}{2 \pi} \int_{-\infty}^{\infty} \hat{M}(k, t) \exp (\mathrm{i} k \chi-\mu|k| y) \mathrm{d} k,
$$

for

$$
\hat{M}(k, t)=\int_{-\infty}^{\infty} M(\chi, t) \exp (-\mathrm{i} k \chi) \mathrm{d} \chi,
$$

where $M(\chi, T)$ is the, as yet undetermined, perturbation to the streamfunction at the outer edge of the shear flow. The two functions, $M$ and $C$, are found by invoking continuity conditions across $y=h(\chi, T)$. Grimshaw \& Yi (1990) note that the appropriate conditions are the continuity of $\psi$ and $\psi_{y}$. This gives

$$
\begin{gathered}
-\frac{1}{2}(h-f)^{2}+C(h-f)=-U h+\frac{1}{2 \pi} \int_{-\infty}^{\infty} \hat{M} \exp (\mathrm{i} k \chi-\mu|k| h) \mathrm{d} k+O\left(\mu^{2}\right), \\
-(h-f)+C=-U-\frac{\mu}{2 \pi} \int_{-\infty}^{\infty} \hat{M}|k| \exp (\mathrm{i} k \chi-\mu|k| h) \mathrm{d} k+O\left(\mu^{2}\right) .
\end{gathered}
$$


Expanding the integrals in powers of $\mu$ shows that

$$
\begin{gathered}
C=h-f-U-\mu \mathscr{B}\{M\}+O\left(\mu^{2}\right), \\
M=U f+\frac{1}{2}(h-f)^{2}+O(\mu),
\end{gathered}
$$

where

$$
\mathscr{B}\{M\}=\frac{1}{2 \pi} \int_{-\infty}^{\infty} \hat{M}|k| \exp \mathrm{i} k \chi \mathrm{d} k=\frac{1}{\pi} \frac{\partial}{\partial \chi} f_{-\infty}^{\infty} \frac{M(y)}{\chi-y} \mathrm{~d} y,
$$

and the Hilbert integral in the alternative form for $\mathscr{B}$ is a Cauchy Principal Value. Perturbations to the undisturbed current width, $A$, and to the exterior velocity, $\Delta$, are defined as

$$
A=h-f-1, \quad \Delta=U-1 .
$$

Then from (2), neglecting terms of $O\left(\mu^{2}\right)$ and an irrelevant constant in $M, A$ satisfies the evolution equation

$$
A_{T}+\frac{\partial}{\partial \chi}\left\{\Delta A-\frac{1}{2} A^{2}+\mu \mathscr{B}\{M\}(1+A)\right\}=0,
$$

where

$$
M=(\Delta+1) f+A+\frac{1}{2} A^{2} .
$$

In Part 1 it was shown that in the hydraulic limit $(\mu \rightarrow 0)$ only supercritical and subcritical flow regimes exist for weakly nonlinear waves. In the limit $\mu \rightarrow 0$ the finite-amplitude equation (9) becomes

$$
A_{T}=\frac{\partial}{\partial \chi}\left\{-\Delta A+\frac{1}{2} A^{2}\right\}+O(\mu) .
$$

There is no forcing and the initial conditions are simply swept upstream or downstream. Results in the remainder of this paper will be presented for $\mu=0.2$. Results for larger values of $\mu$ are qualitatively similar and Part 1 shows that for smaller $\mu$, and in particular $\mu \rightarrow 0$, any near-critical regimes must vanish. For a particular cape the flow then depends on the two parameters $\Delta$, the flow speed of the undisturbed current at the wall, and $\epsilon$, the amplitude of the topography. Note that if $\Delta=-1,(9 b)$ reduces to

$$
M=A+\frac{1}{2} A^{2},
$$

and again the forcing disappears. Descriptions of the evolution of the unforced equation with $\Delta=-1$ can be found in Grimshaw \& Yi (1990).

Equation (9) with an appropriate initial condition is solved here using an adaption of the pseudospectral method of Fornberg \& Whitham (1978), the method used by Grimshaw \& Yi (1990) for the unforced version of this equation. The accuracy of this method for the Korteweg-de Vries equation is discussed by Canuto et al. (1988). Numerical considerations related to the application of this scheme are discussed in the Appendix. 


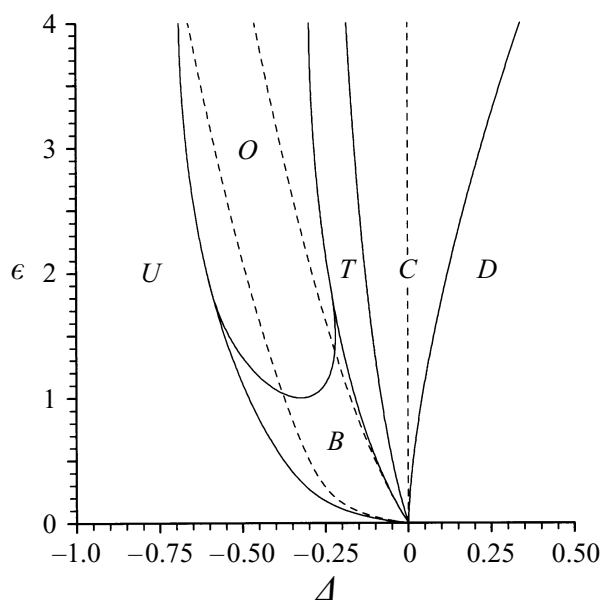

FIGURE 1. A classification of the fully nonlinear flows of (9) with the initial condition (13) and the topography (12). The supercritical regime is denoted by $D$, the transition regime by $T$, the critical regime by $C$, the blocked regime by $B$, the overturning subregime of the blocked regime by $O$ and the subcritical regime by $U$. The dashed lines give the corresponding regime boundaries for the weakly nonlinear $(\epsilon \ll 1)$ flows of Part 1 .

\section{Flow regimes for a symmetric obstacle}

In this section solutions of (9) for the topography

$$
f=\frac{\epsilon}{\chi^{2}+1},
$$

and the initial condition

$$
A(\chi, 0)=-\min (f(\chi), 1)
$$

are considered. This initial condition is equivalent to the condition used in Part 1 of the topography impulsively thrust into a previously uniform shear layer. The effect of changing the initial condition will be investigated later. As this is the same topography and initial condition as considered in Part 1 , it should be expected that the results of Part 1 apply for small amplitude. A classification of the numerical solutions as functions of $\epsilon$ and $\Delta$ is shown in figure 1. For other values of $\mu$ the regime diagram would be qualitatively similar, with only the size of the regimes changing, while for other shapes the position of the regimes may change. In the limit of small $\epsilon$ the regimes are in good agreement with the weakly nonlinear results; however, whereas there are four identifiable regimes in the weakly nonlinear limit, there appear to be five distinct regimes for finite-amplitude flows. Three of these, the subcritical, supercritical and blocked regimes, correspond to regimes found in the weakly nonlinear limit. The blocked regime has an overturning subregime, which is described in more detail later. The fourth weakly nonlinear regime, the steady-critical regime, now splits into a critical regime, which exhibits partial blocking, and a transition regime between the critical and blocked regimes.

Solutions for the subcritical and supercritical regimes are not shown, as they are broadly similar to those in Part 1 . In the supercritical regime the initial wave is swept downstream and evolves into an undular bore and rarefaction governed by the unforced version of (9), the equation of Grimshaw \& Yi (1990). In the vicinity of the forcing a localized steady symmetric wave evolves at large time. This localized wave 
(a)

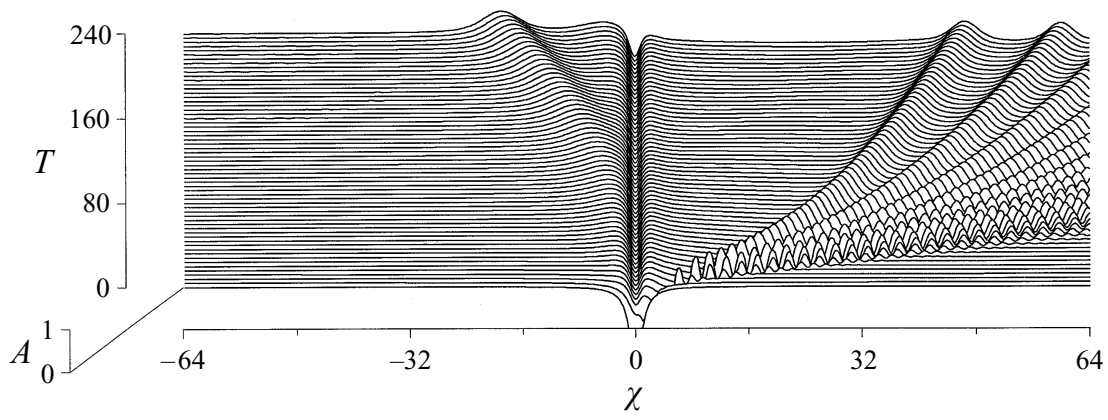

(b)

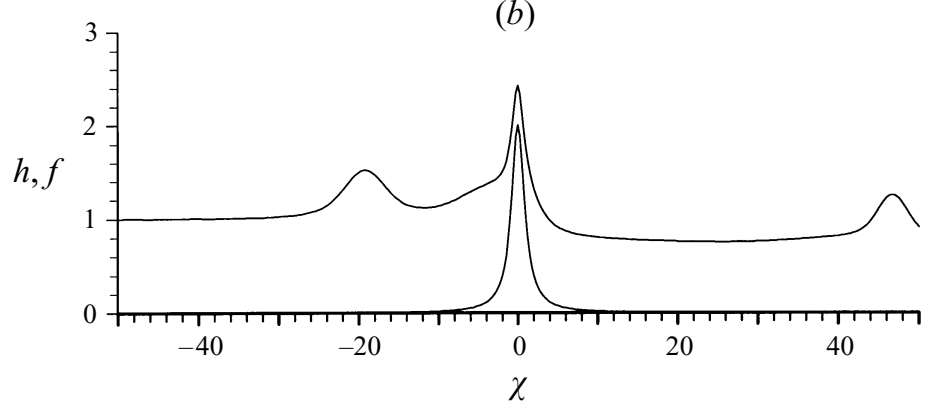

FIGURE 2. A critical solution of (9) with the initial condition (13), the topography (12) and $\epsilon=2$, $\Delta=0$. (a) An evolution diagram of $A$, and $(b)$ a plot of the interface, $h=1+A+f$, and topography, $f$, at $T=240$. In both figures only the central portion of the solution domain is shown.

has the effect of smoothing the shape of the interface, such that the perturbation from $h-1$ is longer but of smaller amplitude than $f$. In the subcritical regime the initial wave is swept upstream and at long time a lee-wave train evolves in the vicinity and downstream of the forcing. Small-amplitude waves are radiated from the upstream edge of the topography as described in the large-amplitude subcritical subregime of Part 1. When $\Delta=-1$ the forcing vanishes and there is no lee-wave train. For $\Delta<-1$ similar behaviour occurs as for $\Delta>-1$, except for a change in the sign of the forcing.

Figure 2 gives a characteristic example of flows in the critical regime. The most significant differences between this solution and the weakly nonlinear solutions is the presence of upstream influence and that the finite-amplitude solution is unsteady at large times. At these times a large-amplitude solitary wave can be seen propagating upstream from the topography, with a smaller-amplitude wave forming at the topography. Downstream of the topography a narrowing of the current width, or shelf, forms, terminated by an undular bore. Since the minimum amplitude of this shelf is always significantly greater than -1 , the cape partially blocks the oncoming rotational fluid. As $\epsilon$ increases the degree of this blocking increases although the rotational fluid is never completely blocked. At large times it could be expected that the solution will consist of a shelf downstream of the topography extending to infinity and a continuous train of solitary waves being formed at the upstream edge of the topography and propagating upstream. These solutions have the same characteristics as the large-cape contour-dynamical solutions of Stern (1991). Figure 2(b) shows that as well as the solitary waves forming at the upstream edge and a shelf downstream, the interface hugs the cape in agreement with Stern's solutions. 
(a)

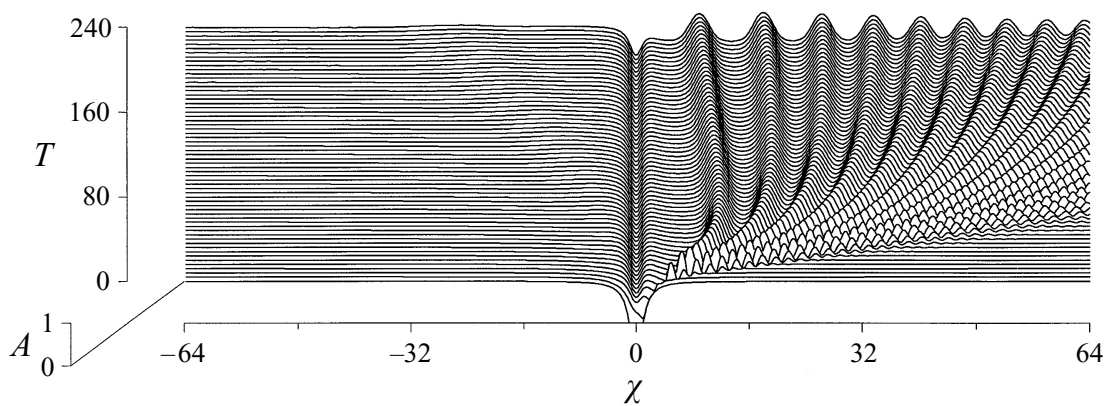

(b)

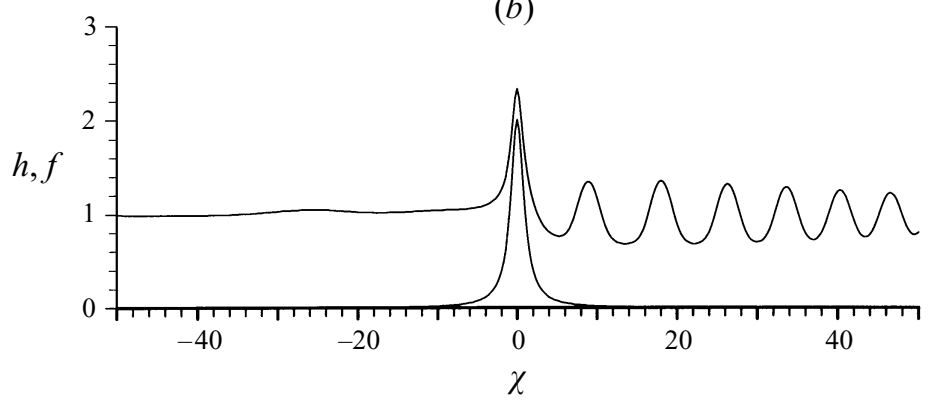

FigURE 3. As for figure 2, except in this case a transition solution is shown with $\Delta=-0.2$.

Figure 3 shows the characteristic behaviour in the transition regime. Downstream the undular bore formed from dispersion of the initial condition initially propagates away. At large time the crest closest to the cape reverses direction and slowly moves upstream. As it propagates upstream its amplitude slowly decreases. Simultaneously a small-amplitude solitary wave propagates away from the upstream edge of the topography creating weak upstream influence; this is clearest in figure 3(b). At even larger times the second wave in the downstream wavetrain turns to propagate upstream, and a second small-amplitude solitary wave propagates away from the upstream edge of the topography. The interface again hugs the cape. Numerical limitations preclude longer integrations; however it appears that once the downstream nonlinear waves reach a critical amplitude they reverse direction and propagate upstream, decaying in the process. As they near the cape small-amplitude solitary waves form on the upstream side and propagate upstream. Thus although waves do not pass over the topography, there is a flux of vorticity from the downstream wavetrain to the upstream solitary waves.

On the line $\Delta=-1$ there is no forcing in (9) and so this line falls into the subcritical regime. A corollary of this is that independently of $\mu$ and the topographic shape, at large $\epsilon$ the subcritical limit of the blocked regime approaches a constant value of $\Delta$ strictly greater than $\Delta=-1$.

The first example of behaviour in the blocked regime, figure 4, falls into the lower part of this regime and, as expected, is similar to the large-amplitude behaviour of the weakly nonlinear solutions. Large-amplitude waves are initially radiated upstream of the obstacle, a steady structure forms at the obstacle and downstream a shelf forms, terminated by an undular bore. At large time the solution will consist of smallamplitude waves being radiated from the upstream edge of the obstacle, a steady 


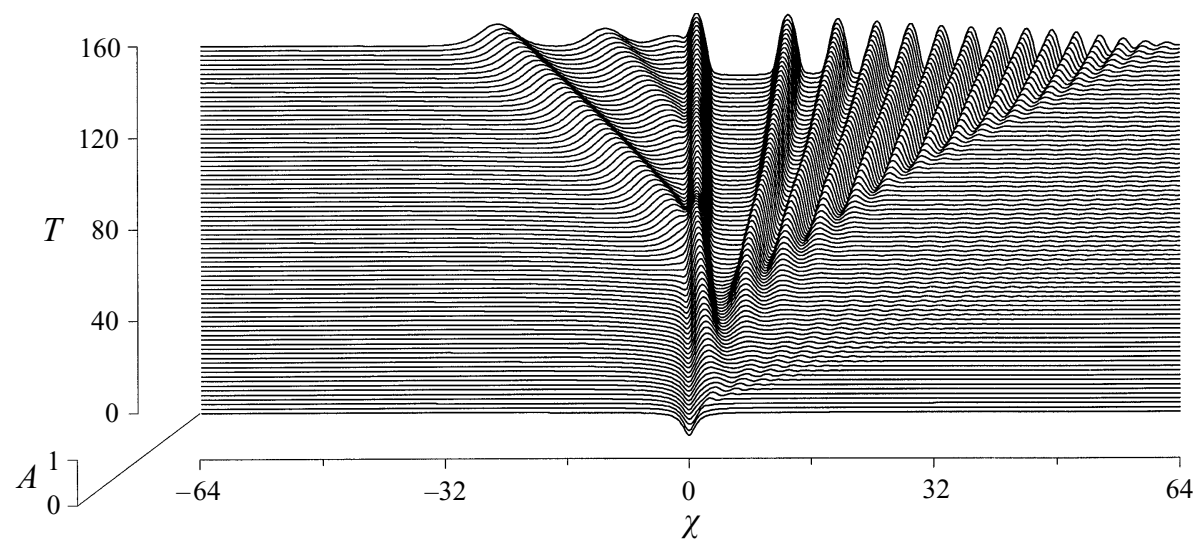

FIGURE 4. A blocked solution of (9) with the initial condition (13), the topography (12), and $\Delta=-0.25, \epsilon=0.5$. Only part of the solution domain is shown.

large-amplitude feature at the obstacle and a constant-width shelf downstream of the obstacle. In this example the amplitude of the shelf is approximately -0.7 , showing significant blocking of the upstream rotational fluid, much stronger than in an equivalent integration for $\Delta=0$.

Two examples of overturning flows in the blocked regime are shown in figure 5. The first, figure 5(a), falls onto the boundary of the overturning subregime, while figure $5(b)$ falls in the interior of this subregime. Qualitatively both these solutions are similar to that shown in figure 4, except that the shelf is of approximately constant amplitude and the minimum is less than the physically realizable critical amplitude of -1 . In both cases instabilities can be seen to form at the upstream end of the shelf. Closer examination reveals that as the shelf evolves (at $T \approx 70$ in figure $5 a$ and $T \approx 50$ in figure $5 b$ ) the derivative of the amplitude becomes large on the downstream side of the standing wave that forms at the cape. The derivative remains large on the downstream side of the standing wave and a cusp-like feature forms to connect the standing wave and the shelf. The numerical scheme fails to resolve this cusp, causing instabilities that propagate downstream on the shelf.

The large derivative and cusp point to overturning of the wave and, ultimately, to the formation of eddies. Once overturning occurs the solution is multivalued and cannot be resolved using the current numerical scheme. Fourier filtering techniques allow solutions beyond the overturning point, as shown in figure 5; however the accuracy of these solutions needs to be tested independently. Contour-dynamical integrations, as well as restoring the short-wave effects, allow the interface to be followed beyond overturning. A detailed investigation of these overturning regimes for arbitrary $\mu$ using contour-dynamical integrations like those of Stern (1991) can be found in Clarke \& Johnson (1997b) together with comparisons between the present nonlinear small- $\mu$ solutions and arbitrary- $\mu$ solutions.

\section{Flow past asymmetric obstacles}

To this point and in Part 1 only solutions for flow past the symmetric topography (12) have been considered. This topography can be generalized to

$$
f=\frac{\epsilon}{\theta^{2}+1},
$$


(a)

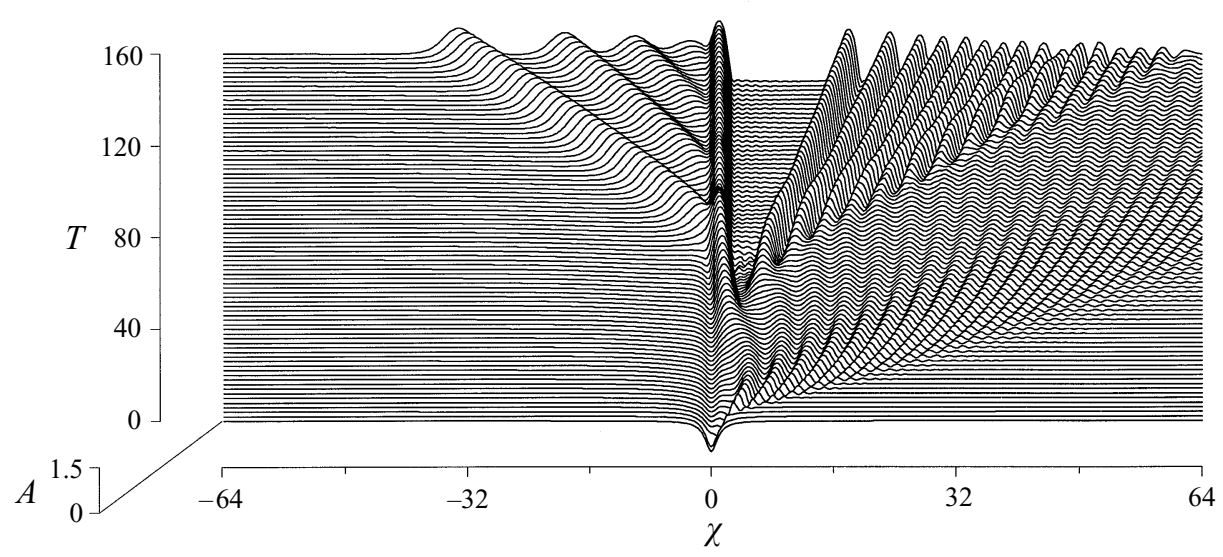

(b)

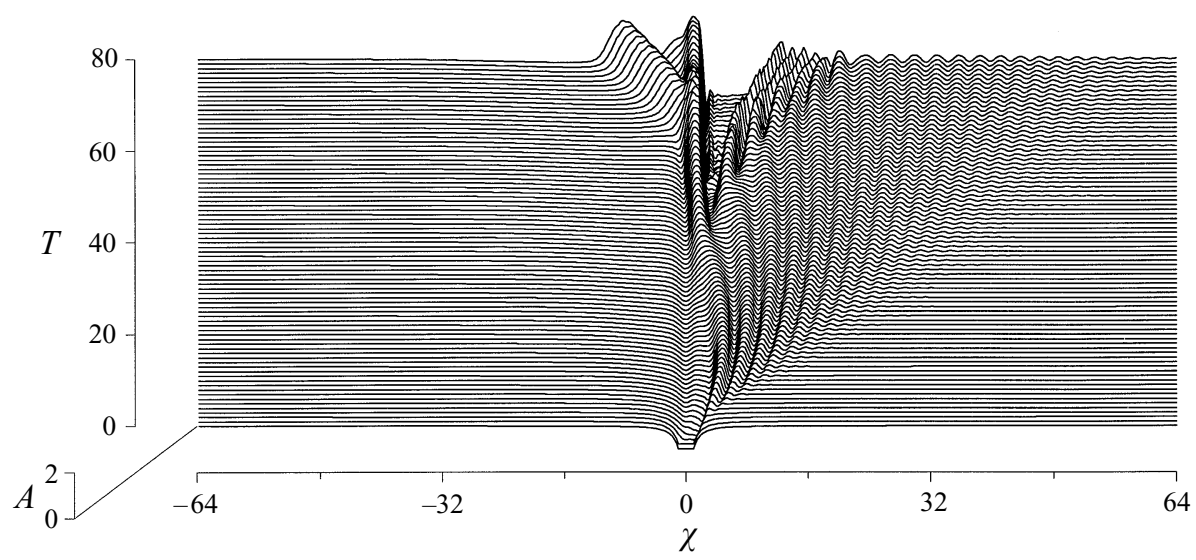

FIGURE 5. Two solutions from the overturning subregime of (9) with the initial condition (13) and the topography (12): (a) $\Delta=-0.3$ and $\epsilon=1$, and (b) $\Delta=-0.4$ and $\epsilon=2$. Only part of the solution domain is shown.

where

$$
(\theta-\chi)\left(\theta^{2}+1\right)+\sigma=0 .
$$

With $\sigma=0$ topography (12) is recovered, for $\sigma<0$ forward facing or 'windward' topography is obtained and when $\sigma>0$ backward facing or 'leeward' topography is obtained. The degree of asymmetry increases with $|\sigma|$ and for $|\sigma|=\sqrt{3}$ the function $f$ is multivalued. A representative example each of flow past windward and leeward topography for the initial condition (13) is shown in figure 6. Apart from the value of $\sigma$ in these solutions, the parameters are identical to the critical solutions of figure $2(a)$.

The general features of the flow shown in figure $6(a)$ are qualitatively similar to those in figure $2(a)$ and so this flow lies in the critical regime. The features of the solution are more accentuated than in figure $2(a)$ due to the severe slope on the upstream face, which suggests that the solution lies further to the right of the critical 
(a)

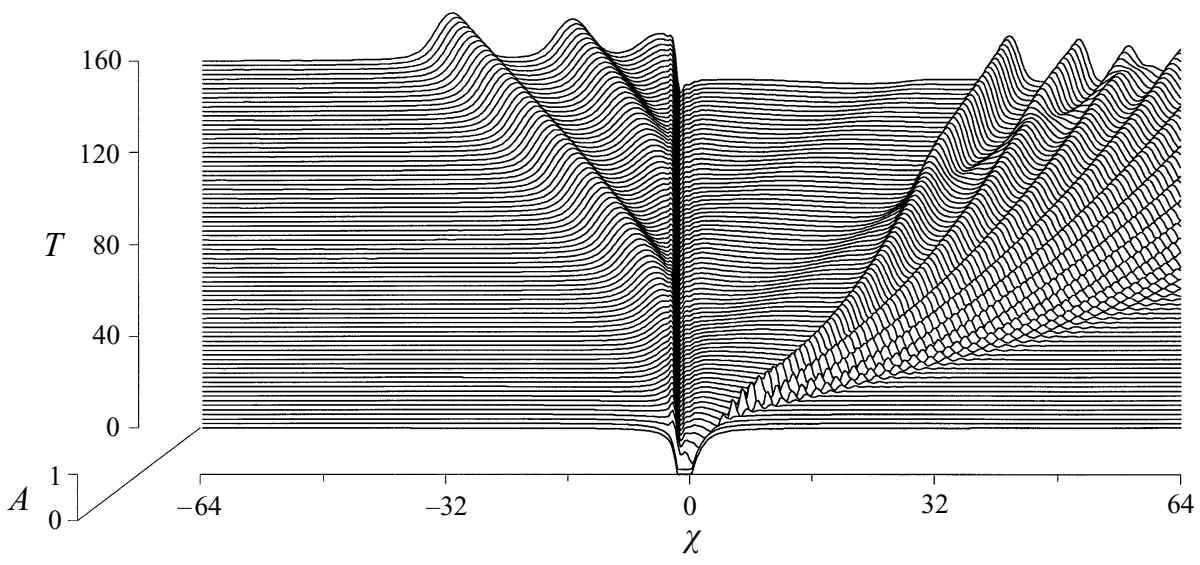

(b)

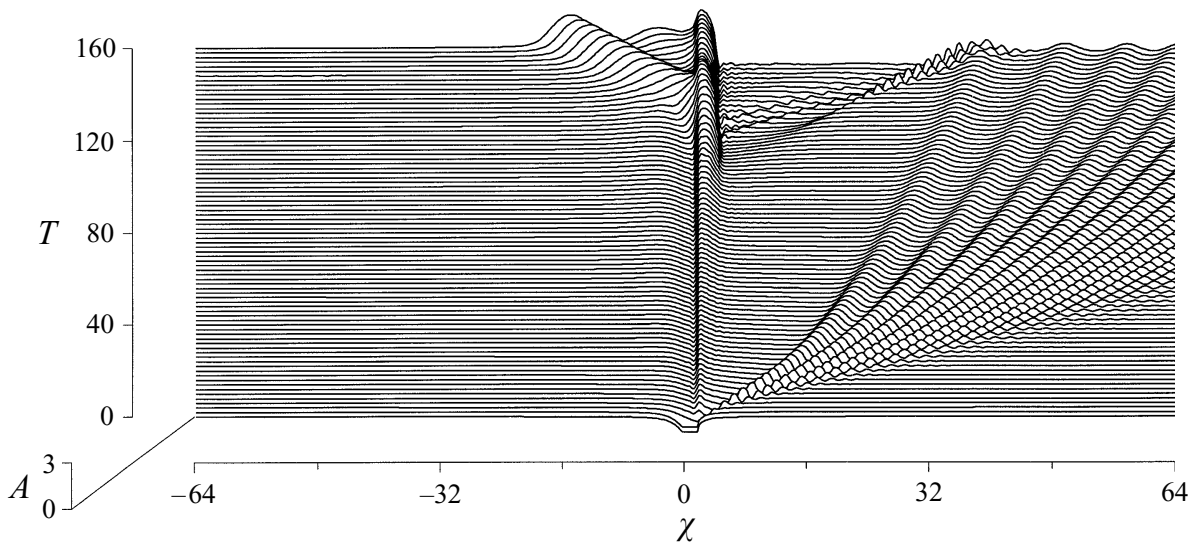

FIGURE 6. Two solutions of (9) with the initial condition (13) for flow past the asymmetric topography (12). Apart from the topography in both figures the parameters are the same as for figure $2(a)$. (a) Flow past a windward-facing obstacle with $\sigma=-1.5$ and $(b)$ flow past a leeward-facing obstacle with $\sigma=1.5$.

regime for this geometry. The significantly larger solitary waves radiated upstream cause complementary waves to be radiated downstream on the shelf.

Figure $6(b)$ shows another example of blocked flow for which the amplitude of the downstream shelf is permanently less than the critical value of -1 , and therefore overturning of the rotational fluid appears likely. The flow is qualitatively similar to figure $5(b)$, except that the leading wave of the initial downstream undular bore does not reverse direction to become trapped near the topography. Instead, a largeamplitude positive disturbance forms, grows under the continuing forcing and then propagates upstream. This disturbance forms because the slope on the downstream face of the topography is sufficiently large to force the flow and overcome advection.

For given flow parameters leeward topography is more likely to generate blocked and overturning flows. In an equivalent figure to figure 1 for leeward topography the transition regime between the blocked and critical regimes would be further to the right. For $\sigma=1.5$ figure $6(b)$ demonstrates that this regime lies to the right of the line $\Delta=0$. The boundary between the critical and supercritical regimes, which 
is controlled by the amplitude of the waves produced on the upstream side of the obstacle, will be moved to the left. Conversely windward topography is less likely to cause blocked and overturning flows and the boundary between the blocked and critical regimes would be further left, while the boundary between the blocked and subcritical regimes will move to the right. The steeper slope on the upstream face of the obstacle will however accentuate the features of the critical flows.

It can be useful in determining flow regimes for asymmetric topography to consider two values of $\mu$ : a wavenumber associated with the upstream side of the obstacle and one associated with the downstream side. As shown in Part 1 the near-critical regimes are due to weak-hydraulic effects, consequently as $\mu$ increases or decreases the size of the regimes also increases or decreases. The size of the blocked regime is controlled by the value of $\mu$ on the downstream face, while the value of $\mu$ on the upstream face controls the size of the critical regime. For a leeward obstacle $\mu$ increases on the downstream side and decreases on the upstream side, so the blocked band increases in size and the critical band decreases. For a windward obstacle $\mu$ increases on the upstream side and decreases on the downstream side, so the critical band increases in size and the blocked band decreases.

Lilly \& Klemp (1979) considered nonlinear non-rotating stratified flow over asymmetric mountains and found that wave amplitude and drag were significantly enhanced for flow over backward-facing mountains and reduced for flow over forwardfacing mountains. They ascribed this to nonlinear wave steepening always occurring on the lee side of the mountain. The present flow is more complicated. Simulations for various $\sigma$ with $\epsilon$ and $\Delta$ fixed show that for large positive $\sigma$ flows are blocked. As $\sigma$ decreases through some small positive value, $\sigma_{c}$ say, wave amplitudes decrease and there is a transition region with flows like those is figure 3. For $\sigma<\sigma_{c}$ flows are critical, and wave amplitudes increase again as $\sigma$ decreases from $\sigma_{c}$. Examining figures 2(a), 6(a) and $6(b)$ show that $6(b)$ is blocked flow in $\sigma>\sigma_{c}$ and $6(a)$ and $2(a)$ are critical flows in $\sigma<\sigma_{c}$ with $6(a)$ further from $\sigma_{c}$ showing larger amplitude waves.

\section{Sensitivity to initial conditions}

The initial condition (13) is simple and corresponds to a physically realizable starting condition (in the laboratory, if not the ocean). However, other choices for the initial condition are possible. Stern (1991) used a number of different initial conditions for his simulations. For $\epsilon>1$ these conditions meant that the interface was initially moved away from the obstacle. It is thus of value to use the present integration method to examine the effect of differing initial conditions on the flow evolution. The integrations above show that in both subcritical and supercritical flows initial disturbances are advected away from the topography and the solution evolves independent of the exact initial flow path. Thus it is only in the blocked and critical regimes that the initial flow can significantly affect the evolution.

If $\Delta=-1$ equation (9) has the solution $A \equiv 0$. Hence, an initial condition that would appear to be a natural alternative to (13) is

$$
A(\chi, 0)=0 .
$$

In this case the vorticity interface is initially displaced an amount equal to the width of the topography. Figure 7(a) shows the evolution of a flow with this initial shape. Apart from the changed initial condition the flow parameters are precisely those for figure $2(a)$. The flow, however, falls into the overturning subregime of the blocked regime. Consequently it appears that eddies will form downstream of the cape. An 
(a)

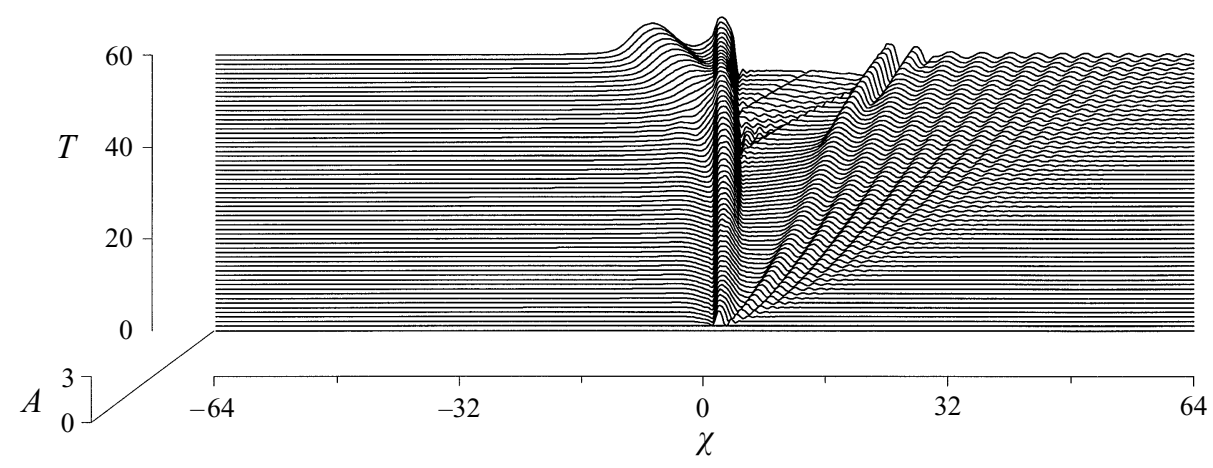

(b)

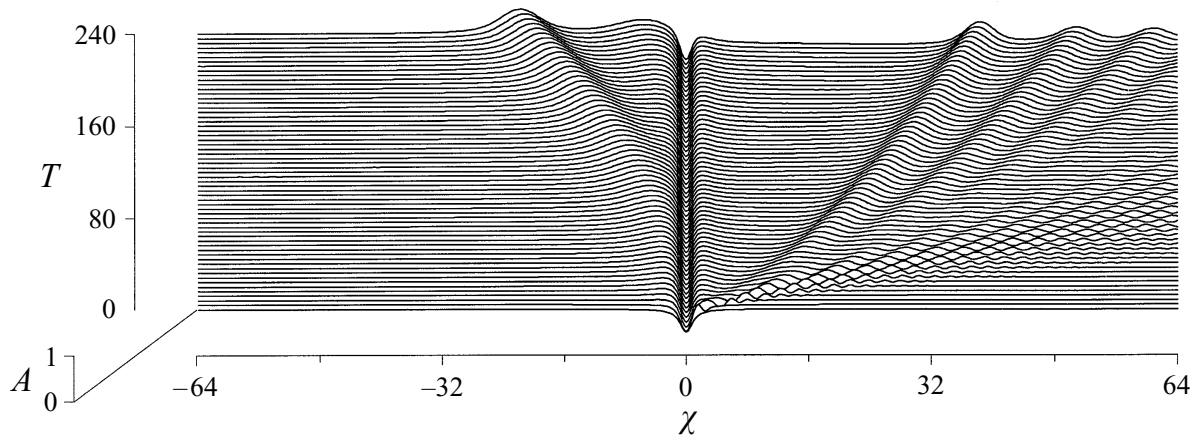

Figure 7. Two solutions of (9) with the topography (12). Apart from the initial condition the parameters are the same as for figure 2(a). (a) The trivial initial condition (15) and $(b)$ an intermediate initial condition (16).

overturning solution forms in this case as the initial forcing creates a positive $(A>0)$ disturbance on the downstream side of the obstacle. Once this happens advection and forcing combine to overcome dispersion and a constant-amplitude shelf and cusp form. This contrasts with the evolution in figure 2(a), where a negative disturbance initially forms on the downstream side of the obstacle. Advection and dispersion balance forcing and a quasi-steady solution forms on the downstream side of the obstacle; large-amplitude positive disturbances form only on the upstream side.

An intermediate initial condition between (13) and (15), but closer to (15), is

$$
A(\chi, 0)=-\min \left(\frac{1}{4} f(\chi), 1\right) .
$$

The evolution of a flow with this initial condition, but otherwise identical to figures $2(a)$ and $7(a)$ is shown in figure $7(b)$. The flow at large times is identical to that in figure $2(a)$. Here the only effect of changing the initial condition from (13) is to reduce the initial transients. It appears that for this geometry a critical amount of vorticity must be initially present near the outcrop to cause blocking or overturning.

The flow evolutions of figure 7 and figure $2(a)$ suggest that within the near-critical regimes for a given geometry and exterior flow there is a discrete number of longtime configurations with the path to each configuration depending solely on the initial condition. In the examples here and other related simulations only two such longtime configurations have been found: critical flows and blocked or overturning flows. 
Although Stern (1991) varied the initial conditions widely for his $\Delta=0$ simulations it appears that none of them supplied sufficient vorticity near the outcrop to create an overturning flow.

\section{Conclusions}

This paper has investigated the generation of finite-amplitude long waves by the flow of a sheared current past topography to obtain results complementary to the weakly nonlinear results of Part 1. There the flows fall into one of four broad regimes: supercritical, steady critical, blocked and subcritical. It is the steady-critical and blocked regimes that are of particular interest here, as these can cause blocking and overturning of the upstream fluid. For finite-amplitude flows the steady-critical regime divides into a critical regime and a transition regime, both of which exhibit partial blocking of the upstream rotational fluid. In the critical regime the long-time solution consists of a negative shelf downstream of the cape and positive upstream influence with unsteady nonlinear waves generated at the upstream edge of the cape. In the transition regime the long-time solution is a quasi-steady nonlinear wavetrain superimposed on a negative shelf downstream of the obstacle and a weak positive upstream influence with unsteady waves again generated at the upstream edge of the cape. For small capes in the blocked regime the long-time flow consists of a large-amplitude standing wave at the cape, a negative shelf downstream and strong upstream influence with unsteady waves generated at the upstream edge. As the amplitude of the cape increases the degree of blocking increases until the amplitude of the downstream shelf decreases below the critical value of -1 . For amplitudes greater than this the standing waves at the cape and shelf are joined by a cusp-like feature, indicating in practice that the interface has overturned.

It has been argued here, following the results of Part 1, that the blocked and critical regimes are due to weak-hydraulic effects. Thus the size of the blocked and critical bands should decrease as the topographic lengthscale $\mu^{-1}$ lengthens. Three results support this. First, in the limit $\mu \rightarrow 0$ the forcing vanishes and (9) reduces to the nonlinear advection equation. Secondly, for small topography the limits of the critical and blocked regimes agree with the weakly nonlinear results and for large topography these limits do not diverge markedly from the continuation of the weakly nonlinear results. Finally, the solutions for flow past asymmetric topography where the characteristic lengthscales on each side of the obstacle differ show that the extent of each regime increases and decreases as predicted by weak-hydraulic effects.

The flow regime diagram, figure 1, applies to the particular cape (12) and initial condition (13). It has been shown, however, that changes in either the cape profile or initial condition can have a dramatic effect on the evolution of the flow and its form at large times. Blocking and overturning are caused by nonlinear steepening downstream of the cape and so form more readily in flow past leeward-facing topography. Critical flows occur when nonlinear waves form on the upstream side of the cape and so occur more readily in flow past windward-facing topography. Increasing the initial vorticity near a cape sufficiently can cause blocked or overturning flows in regions where critical flows might otherwise be expected. Integrations with different initial conditions show that multiple long-time flows are possible for (9).

In Part 1 it is noted that to investigate flows with possible weak-hydraulic transitions it is valuable to consider initial conditions with upstream flow reversals (non-zero $\Delta$ ). However, the results for asymmetric capes and differing initial conditions show that 
both types of near-critical flows, blocked and critical, identified for $\Delta \neq 0$ can occur in flows which are initially unidirectional with $\Delta=0$.

The flow geometry has been chosen here to allow some comparison with Stern (1991). Most of Stern's solutions correspond to $\Delta=0$, although Stern also considered the effect on these flows of introducing a small amount of vorticity into the outer fluid and adding a constant along-shore velocity. No direct comparisons can be made with the vorticity-containing outer flows, but the constant along-shore velocity corresponds to simply changing $\Delta$. Care must also be taken in comparing the present flows with Stern's, as his simulations used a range of initial conditions that fall between (13) and (15) and as seen in $\S 5$ the choice of initial condition has important consequences on the long-time flow. Stern's $\Delta=0$ solutions correspond to flows with $\epsilon=1,2$ and 3 here. For $\epsilon=1$ his solutions are taken to $t=65$ only, which in our notation is $T=O(10)$. Figures 2 and 5 show that considerably longer times are needed to determine the final state of the flow. For $\epsilon=2$ Stern's solutions show a constant height shelf downstream, but with the width of the current approximately half the width of the original current indicating partial blocking of the flow. Upstream of the cape a large-amplitude solitary wave is forming. In figure 2 the same characteristic features can be observed, although the blocking is not as great. For $\epsilon=3$ his solutions show complete blocking of the upstream flow and an upstream solitary wave which has almost propagated away from the obstacle. Stern's solutions thus fall into what we have described here as the critical regime.

Finally, Stern found that for $\epsilon=3$ when $\Delta$ (his $W$ ) was increased to 0.25 only partial blocking occurred and when $\Delta$ was further increased to 1.5 no blocking occurred. This is consistent with our results. When $\Delta=0.25$ the flow falls into the critical regime and when $\Delta=1.5$ into the supercritical regime. Stern notes "Thus there is a critical $W$ (between 0.25 and 1.5), such that all influences are propagated downstream, and no blocking occurs." Our study indicates that this critical value occurs much closer to $W=0.25$ than to $W=1.5$.

This work was carried out under grant number GR3/09174 from the Natural Environment Research Council. S.R.C. would like to thank the London Goodenough Trust for Overseas Graduates for helping make his stay in London possible.

\section{Appendix. Numerical considerations}

When $\Delta$ is large and negative lee waves are generated by the flow past the topography. Balancing the linear terms in (9) gives an estimate of the wavenumber as

$$
k_{c}=|\Delta| / \mu .
$$

The resolution of the spectral method is given by the Nyquist critical frequency. Matching these two frequencies shows that accurately resolving the lee waves requires

$$
\Delta \chi=\frac{L}{N} \leqslant \frac{\pi \mu}{|\Delta|} .
$$

In the simulations presented here $\Delta \chi=0.25$. For KdV-like equations, where a thirdorder derivative replaces the Hilbert transform term, $k_{c}=O\left(|\Delta|^{1 / 2}\right)$ and such fine resolution as $\Delta$ is increased in magnitude is not needed.

For flows in which overturning does not occur a Fourier filter was applied to the solutions every $T_{s m}$ time units to prevent numerical instabilities. The most effective filter, damping instabilities while maintaining the significant features of the solution, 
was found to be the sharpened raised cosine filter (Canuto et al. 1988) with typically $T_{s m}=2$. An estimate of the effect of the filtering can be found by considering the similar raised cosine filter, which Canuto et al. (1988) state is equal to second-order viscosity with the coefficient

$$
v=\frac{(\Delta \chi)^{2}}{4 T_{s m}} .
$$

For a typical integration, where $\Delta \chi=0.25$, this gives $v<0.01$.

As argued in $\$ 3$ once overturning occurs the simulations using (9) are no longer valid. However, for the purpose of analysing the results these can proceed up to and past the overturning time using selective derivate filtering in conjunction with smoothing every $T_{s m}$ time units. Experience showed that the term leading to the strongest instabilities when calculating the spatial derivatives of (9) was the Hilbert transform

$$
\mathscr{B}\{M\}=\mathscr{F}^{-1}\{|k| \mathscr{F}\{M\}\},
$$

where the operators $\mathscr{F}\{\cdot\}$ and $\mathscr{F}^{-1}\{\cdot\}$ represent the forward and inverse Fourier transforms. Therefore this term was replaced by

$$
\mathscr{F}^{-1}\{f(k)|k| \mathscr{F}\{M\}\},
$$

where $f(k)$ is the exponential cutoff filter (Canuto et al. 1988)

$$
f(k)= \begin{cases}1 & \text { if } \quad k<k_{\sigma} \\ \exp \left[-\sigma\left(k-k_{\sigma}\right)^{4}\right] & \text { if } \quad k>k_{\sigma} .\end{cases}
$$

with typically $k_{\sigma}=0.75 k_{\max }$ and

$$
\sigma^{1 / 4}\left(k_{\max }-k_{\sigma}\right) \gg 1
$$

\section{REFERENCES}

Canuto, C., Hussaini, M., Quarteroni, A. \& Zang, T. 1988 Spectral Methods in Fluid Dynamics. Spinger.

Clarke, S. \& Johnson, E. 1997 a Topographically forced long waves on a sheared coastal current. Part 1. The weakly nonlinear response. J. Fluid Mech. 343, 131-151.

Clarke, S. \& Johnson, E. $1997 b$ Eddy generation and long-wave theories for flows along variable coasts. In preparation.

DRITSCHEL, D. 1989 Contour dynamics and contour surgery: numerical algorithms for extended, high-resolution modelling of vortex dynamics in two-dimensional, inviscid, incompressible flows. Comput. Phys. Rep. 10, 77-146.

ForNberG, B. \& Whitham, G. 1978 A numerical and theoretical study of certain nonlinear wave phenomena. Phil. Trans. R. Soc. Lond. A 289, 373-404.

GiLl, A. 1977 The hydraulics of rotating channel flow. J. Fluid Mech. 80, 641-671.

Grimshaw, R. 1987 Resonant forcing of barotropic coastally trapped waves. J. Phys. Oceanogr. 17, 54-65.

Grimshaw, R. \& YI, Z. 1990 Finite-amplitude long waves on coastal currents. J. Phys. Oceanogr. 20, 3-18.

Grimshaw, R. \& YI, Z. 1992 Resonant generation of finite-amplitude waves by flow past topography on a $\beta$-plane. Stud. Appl. Maths 88, 89-112.

Haynes, P., Johnson, E. \& Hurst, R. 1993 A simple model of Rossby-wave hydraulic behaviour. J. Fluid Mech. 253, 359-384.

Hughes, R. 1985 a Multiple criticalities in coastal flows. Dyn. Atmos. Oceans 9, 321-340. 
Hughes, R. $1985 b$ On inertial currents over a sloping continental shelf. Dyn. Atmos. Oceans 9, 49-73.

Hughes, R. $1986 a$ On the conjugate behaviour of weak along-shore flows. Tellus 38A, 227-284.

Hughes, R. $1986 b$ On the role of criticality in coastal flows over irregular bottom topography. Dyn. Atmos. Oceans 10, 129-147.

Hughes, R. 1987 The role of higher shelf modes in coastal hydraulics. J. Mar. Res. 45, 33-58.

Hughes, R. 1989 The hydraulics of local separation in a coastal current, with application to the Kuriosho meander. J. Phys. Oceanogr. 19, 1809-1820.

LiLly, D. \& KLEMP, J. 1979 The effects of terrain shape on nonlinear hydrostatic mountain shapes. J. Fluid Mech. 95, 241-261.

Stern, M. 1991 Blocking an inviscid shear flow. J. Fluid Mech. 227, 449-472. 